
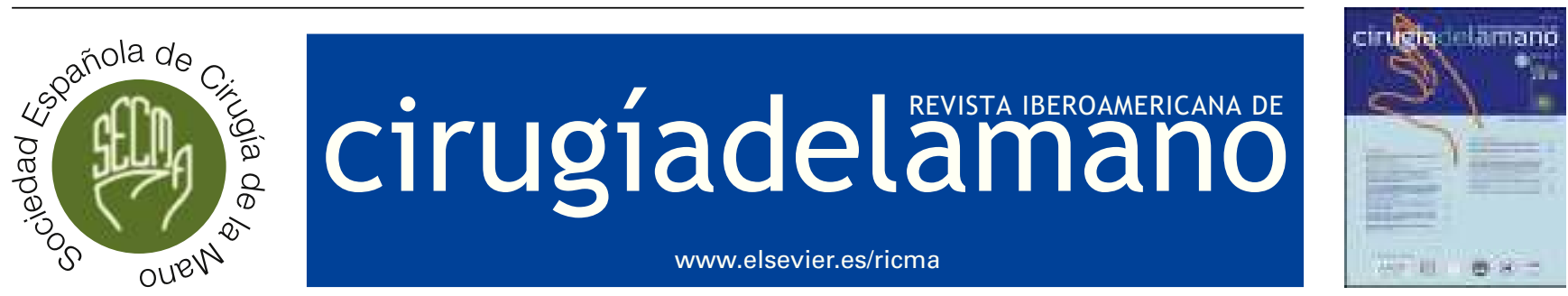

ARTÍCULO ORIGINAL

\title{
Estudio de las unidades neurofuncionales del flexor carpi ulnaris y su utilidad en las transferencias tendinosas
}

\author{
M. Llusá Pérez ${ }^{a, b, *}$, G.J. Tarnawski Español ${ }^{a}$, D. Gutiérrez Medina ${ }^{a}$, \\ J.R. Ballesteros Betancourt ${ }^{a, c}$ y M.R. Morro Martía,
}

a Laboratorio de Macro-Microdisección y Anatomía Quirúrgica, Departamento de Anatomía y Embriología Humana, Facultad de Medicina, Universidad de Barcelona, Barcelona, España

b Servicio de Cirugía Ortopédica y Traumatología, Hospital de Traumatología Vall d'Hebron, Barcelona, España

c Servicio de Cirugía Ortopédica y Traumatología, Hospital Clínic de Barcelona, Barcelona, España

Recibido el 7 de enero de 2016; aceptado el 27 de febrero de 2016

Disponible en Internet el 4 de abril de 2016

\section{PALABRAS CLAVE \\ Inervación; \\ Flexor carpi ulnaris; \\ Tinción de Sihler; \\ Transferencia \\ tendinosa; \\ Anatomía}

\begin{abstract}
Resumen
Objetivos: Este trabajo pretende documentar la inervación intramuscular del flexor carpi ulnaris (FCU), su vascularización, y su consecuente organización en unidades neurofuncionales. Así mismo, se discute la relevancia de dichas unidades en la realización de transferencias tendinosas de este músculo con su tendón dividido. Aunque en algunos centros ya se ha puesto en práctica la idea de dividir el tendón, hay pocos trabajos en la literatura que demuestren fotográficamente el sustrato anatómico subyacente.

Material y métodos: Se tiñeron según la técnica de Sihler 6 FCU extraídos de cadáveres humanos criopreservados. Para estudiar su vascularización, en 5 de ellos se inyectó el árbol arterial con látex negro.

Resultados: En todos los especímenes ambas cabezas del FCU se correspondían con dos unidades neurofuncionales que presentaban una inervación independiente (tipo III de Taylor). Los 5 especímenes inyectados pertenecían a los tipos ॥ y III de Mathes-Nahai.

Conclusiones: La divisibilidad del músculo en 2 unidades neurofuncionales independientes con vascularización e inervación propia permite obtener 2 tendones transferibles a 2 músculos diferentes, optimizándose la transferencia.

(C) 2016 SECMA. Publicado por Elsevier España, S.L.U. Este es un artículo Open Access bajo la licencia CC BY-NC-ND (http: / / creativecommons.org/licenses/by-nc-nd/4.0/).
\end{abstract}

\footnotetext{
* Autor para correspondencia.

Correo electrónico: mllusa@ub.edu (M. Llusá Pérez).
} 


\section{KEYWORDS}

Innervation;

Flexor carpi ulnaris;

Sihler's stain;

Tendon transfer; Anatomy
Study of the neurofunctional units of the flexor carpi ulnaris and their usefulness in tendon transfers

\begin{abstract}
Objectives: The purpose of this paper is to document the intramuscular innervation of flexor carpi ulnaris (FCU), its vascularisation, and its subsequent organisation in neurofunctional units. The relevance of these neurofunctional units in the performance of split-tendon transfers of this muscle is also discussed. Although this idea has already been put into practice in some surgical centres, there are few published works in the literature which graphically show its underlying anatomical basis.

Material and methods: Six FCU specimens were extracted from human cryopreserved cadavers and stained following Sihler's technique. In order to study the vascularization, 5 of them were also injected with coloured latex through the brachial artery.

Results: In all of the specimens, each of the two heads of the FCU corresponded to a neurofunctional unit, and was independently innervated (Taylor's type III pattern). The 5 injected specimens belonged to either type II or type III of the Mathes-Nahai classification.

Conclusions: The divisibility of the muscle into two independently vascularised and innervated neurofunctional units allows two tendons to be obtained from the donor muscle, which are then transferable to two different muscles, thus optimising the transfer.

(C) 2016 SECMA. Published by Elsevier España, S.L.U. This is an open access article under the CC BY-NC-ND license (http://creativecommons.org/licenses/by-nc-nd/4.0/).
\end{abstract}

\section{Introducción}

En el tratamiento de pacientes con déficits neurológicos o funcionales del aparato locomotor, un conocimiento anatómico exhaustivo y actualizado es imprescindible. El presente trabajo se centra en la descripción de las unidades neurofuncionales del músculo flexor carpi ulnaris (FCU) y sus aplicaciones en el diseño de transferencias tendinosas.

Definimos como una unidad neurofuncional a un conjunto de fibras dentro de un músculo que conforman una estructura anatómica distinta y son inervadas por un mismo ramo nervioso, lo que implica que son elementos con una identidad morfológica y fisiológica propias. La idea de que el músculo esquelético presenta una compartimentalización funcional cuenta ya con varias décadas de historia; esta compartimentalización se ha definido tradicionalmente en función de la inervación, correlacionándola con la organización somatotópica del SNC ${ }^{1}$. Por otra parte, una serie de estudios publicados por Taylor et al. en los años noventa $a^{2,3}$ introdujo el concepto de angiosoma, en referencia a bloques macizos compuestos de diferentes tejidos dependientes de un único «vaso principal» o source vessel. En un trabajo posterior ${ }^{4}$, Taylor et al. describieron la relación de estos angiosomas con la inervación del tegumento y los tejidos profundos subyacentes, lo cual condujo a la definición de «territorios neurovasculares» tributarios de un nervio concreto e irrigados por un vaso principal. El interés quirúrgico de estos estudios radica en la posibilidad de identificar componentes dentro de un mismo músculo que pertenezcan a territorios neurovasculares distintos, y que por lo tanto puedan separarse y funcionar de forma independiente en colgajos y transferencias. Un estudio de Lim et al. arrojó nueva luz sobre este tema describiendo las subunidades funcionales del FCU con el nombre de «compartimentos neuromusculares» combinando la arteriografía y la tinción de Sihler y utilizando principalmente ejemplares de
Macaca fascicularis ${ }^{5}$. Descrita a finales del siglo xix por el alemán Charles Sihler, esta técnica fue olvidada largo tiempo por los anatomistas y recuperada recientemente por algunos investigadores ${ }^{6-9}$, puesto que aún hoy representa un modo excelente de observar la inervación de estructuras, especialmente del aparato locomotor, sin exponerlas a los posibles artefactos de la disección. La técnica permite, mediante la transparentación del tejido muscular, observar «al natural» la distribución intramuscular de los ramos nerviosos, que quedan teñidos con hematoxilina.

Partiendo de un enfoque muy similar y una metodología algo diferente, nuestro trabajo documenta fotográficamente la existencia de 2 unidades neurofuncionales en el FCU humano. Se lleva a cabo el estudio vascular y nervioso simultáneamente, con 2 objetivos principales: primero, identificar los ramos nerviosos para el FCU, y segundo, delimitar las unidades neurofuncionales desde un punto de vista anatómico. Adicionalmente, se discuten las situaciones en las cuales puede ser útil realizar una transferencia tendinosa con el tendón dividido en 2 mitades, correspondientes a cada unidad neurofuncional.

\section{Material y métodos}

Se llevó a cabo una versión modificada de la tinción de Sihler sobre 6 especímenes extraídos de cadáveres humanos criopreservados ${ }^{7}$. Los especímenes provenían de cuerpos donados anónima y voluntariamente al Servicio de Donación de Cuerpos de nuestra institución. Previamente a la fijación, 5 de los especímenes fueron inyectados con látex negro a través de la arteria braquial. Tras la extracción de los FCU de los antebrazos, las piezas se fijaron en formalina al $10 \%$ durante un período de 3 semanas. Acto seguido se sumergieron en una solución acuosa de hidróxido de potasio al $3 \%$ y peróxido de hidrógeno al $0,3 \%$, cuyo objetivo era macerar 


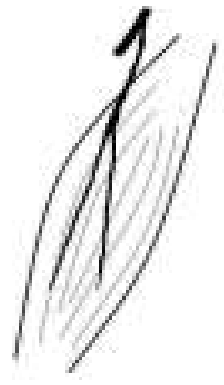

1

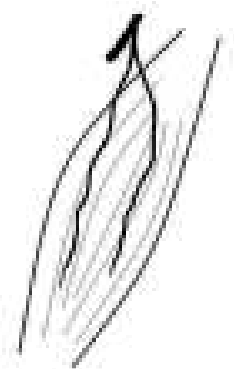

II



III

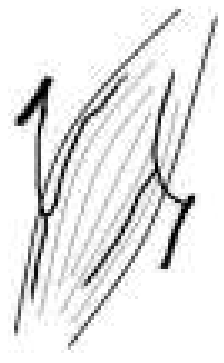

IV

Figura 1 Tipos de inervación, según Taylor et al. ${ }^{4}$. Tipo I: ramo único no ramificado entrando al músculo; tipo ॥: ramo único ramificado entrando al músculo; tipo II: ramos múltiples procedentes de un mismo nervio; tipo ıv: ramos múltiples procedentes de distintos nervios.

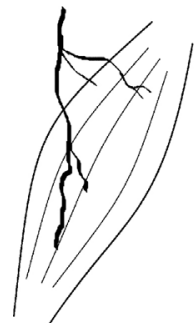

I



II

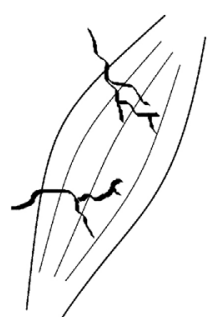

III

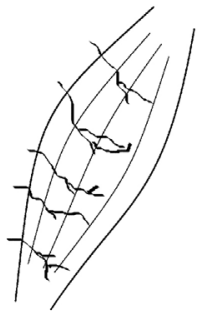

IV

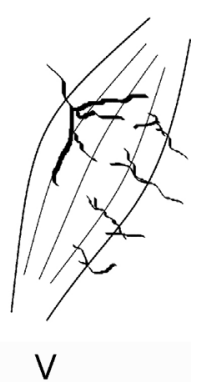

$\mathrm{V}$

Figura 2 Tipos de vascularización, según Mathes y Nahai ${ }^{10}$. Tipo I: pedículo vascular único; tipo II: pedículo vascular dominante y pedículos menores; tipo II: 2 pedículos vasculares codominantes; tipo Iv: pedículos vasculares segmentarios; tipo v: pedículo vascular dominante y pedículos secundarios segmentarios.

la pieza y despigmentarla. La sumersión en hidróxido de potasio y peróxido de hidrógeno se mantuvo durante aproximadamente 4 semanas en función del estado de maceración de la pieza, que era juzgado por su grado de translucidez. La solución fue cambiada cada 2 o 3 días, momento en el que se aprovechó para lavar los especímenes en agua corriente de forma que se desprendieran los residuos generados por el proceso.

Cuando las piezas se consideraron suficientemente maceradas y despigmentadas, se inició una nueva sumersión, esta vez en la solución de detinción Sihler I. Esta se obtiene mezclando hidrato cloral acuoso al $1 \%$, ácido acético glacial puro y glicerina en proporción de 6:1:1 y sirve al propósito de decalcificar el músculo. Esta fase se prolongó durante 3 o 4 semanas. Se controló semanalmente el estado de la pieza, pues al ser un proceso agresivo sobre el tejido se corre el riesgo de destruir las fibras musculares, hecho que también ocurre con la maceración. Tras la decalcificación, los especímenes se sumergieron en la solución Sihler II, consistente en una mezcla de hematoxilina de Ehrlich, glicerina e hidrato cloral acuoso al 1\% en proporción 1:1:6. Los especímenes se mantuvieron sumergidos en esta nueva solución durante al menos 4 semanas más, momento en que se consideraron completamente teñidos.

La fase final del proceso consistió en la colocación de cada músculo en un visor de vidrio transparente equipado con una fuente de luz trasera. El visor fue inundado con la mezcla Sihler I y se mantuvo la pieza sumergida durante $90 \mathrm{~min}$, removiendo cuidadosamente y reemplazando la solución cuando perdía transparencia. Cuando los detalles de la inervación se hicieron visibles de forma óptima por transiluminación, se tomó nota de los hallazgos y se realizaron fotografías de cada estructura siguiendo un protocolo. Se registró el número de ramas que se desprendían directamente del nervio ulnar y se clasificó a cada espécimen según el sistema de Taylor $^{4}$ (fig. 1). También se contabilizaron los ramos intramusculares que discurrían dentro de cada cabeza del músculo. Finalmente, se describió la vascularización en los especímenes inyectados y se los clasificó por su tipo de Mathes-Nahai ${ }^{10}$ (fig. 2). Se decidió no medir las estructuras descritas, dado que la tinción de Sihler puede alterar las dimensiones naturales de los tejidos dando lugar a resultados falsos.

\section{Resultados}

En todos los especímenes las cabezas humeral y ulnar del FCU presentaban una inervación separada. Todos los casos se consideraron tipo III de Taylor, puesto que los ramos musculares (en número de 2 o 3 ) que entraban al músculo emergían directamente del tronco principal del nervio ulnar, y no a partir de un ramo muscular común (fig. 3).

Estos ramos provenientes del nervio ulnar penetraban siempre en el vientre muscular por su tercio proximal, adaptándose rápidamente al eje longitudinal de este y siguiendo un trayecto rectilíneo. En algunos casos, una misma cabeza estaba inervada por 2 ramos intramusculares en vez de uno; esto ocurría más frecuentemente en la cabeza ulnar (fig. 4). No existía en ninguno de los casos ningún tipo de 
Tabla 1 Clasificación de los especímenes según su tipo de vascularización (Mathes-Nahai) e inervación (Taylor)

\begin{tabular}{lllll} 
& $\begin{array}{l}\text { Clasificación de Taylor } \\
\text { (n. }{ }^{\circ} \text { ramos emergentes } \\
\text { del nervio ulnar) }\end{array}$ & $\begin{array}{l}\text { N. }{ }^{\circ} \text { de ramos } \\
\text { intramusculares en } \\
\text { la cabeza humeral }\end{array}$ & $\begin{array}{l}\text { N. }{ }^{\circ} \text { de ramos } \\
\text { intramusculares en } \\
\text { la cabeza ulnar }\end{array}$ & $\begin{array}{l}\text { Clasificación de } \\
\text { Mathes-Nahai }\end{array}$ \\
\hline FCU 1 & III (2) & 1 & 1 & III \\
FCU 2 & III (2) & 1 & 1 & II \\
FCU 3 & III (2) & 1 & 1 & III \\
FCU 4 & III (3) & 1 & 2 & II \\
FCU 5 & III (2) & 2 & 2 & - \\
FCU 6 & III (2) & 1 & 1 & II \\
\hline
\end{tabular}

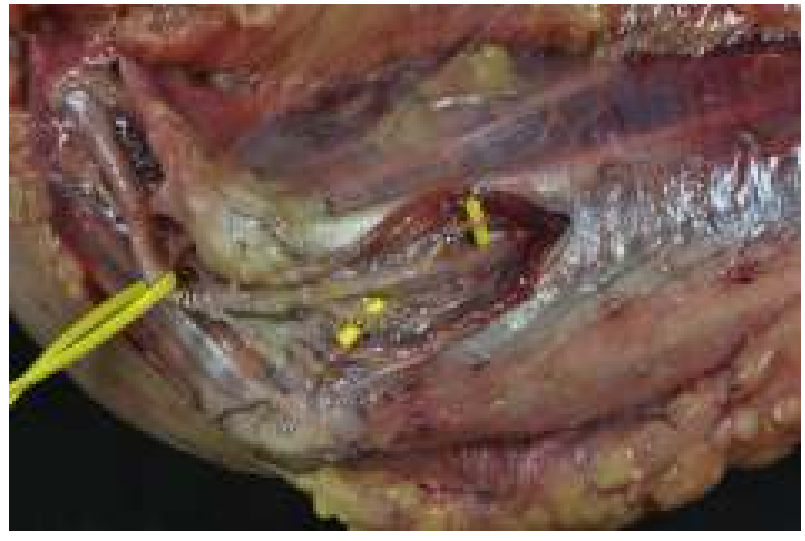

Figura 3 Preparación anatómica que muestra el nervio ulnar en el punto en que se separan sus ramos motores para el FCU.Asteriscos: ramos motores; E: epicóndilo medial (epitróclea); NU: nervio ulnar; O: olécranon.

comunicación nerviosa que cruzara el tendón que separa ambas cabezas. Todas las observaciones quedan detalladas en la tabla 1.

Por lo que respecta a la vascularización del FCU, los 5 especímenes inyectados con látex fueron clasificados dentro del tipo II o III de Mathes-Nahai. Las venas viajaban como satélites de las arterias en el pedículo vascular. Intramuscularmente, los vasos se ceñían notablemente al recorrido trazado por los nervios. Ambas cabezas del músculo estaban adecuadamente perfundidas por pedículos vasculares diferentes.

\section{Discusión}

El objetivo de nuestro trabajo era revisar la inervación del FCU e identificar el sustrato anatómico de las unidades neurofuncionales del FCU. Aunque una de las limitaciones de este estudio es el reducido tamaño de la muestra, la uniformidad de los resultados obtenidos invita a reflexionar sobre algunos detalles morfológicos de relevancia quirúrgica. Llama la atención que a pesar de la existencia de variaciones en el número de ramos nerviosos que emitía el nervio ulnar para el FCU, dichos ramos siempre quedaban limitados a una de las 2 cabezas del músculo, tal y como esperábamos. La clasificación de Taylor está diseñada para correlacionarse con la dificultad técnica que acarrea la división quirúrgica de los compartimentos, siendo el tipo । el más complejo y el tipo ıv el más sencillo. La pertenencia de

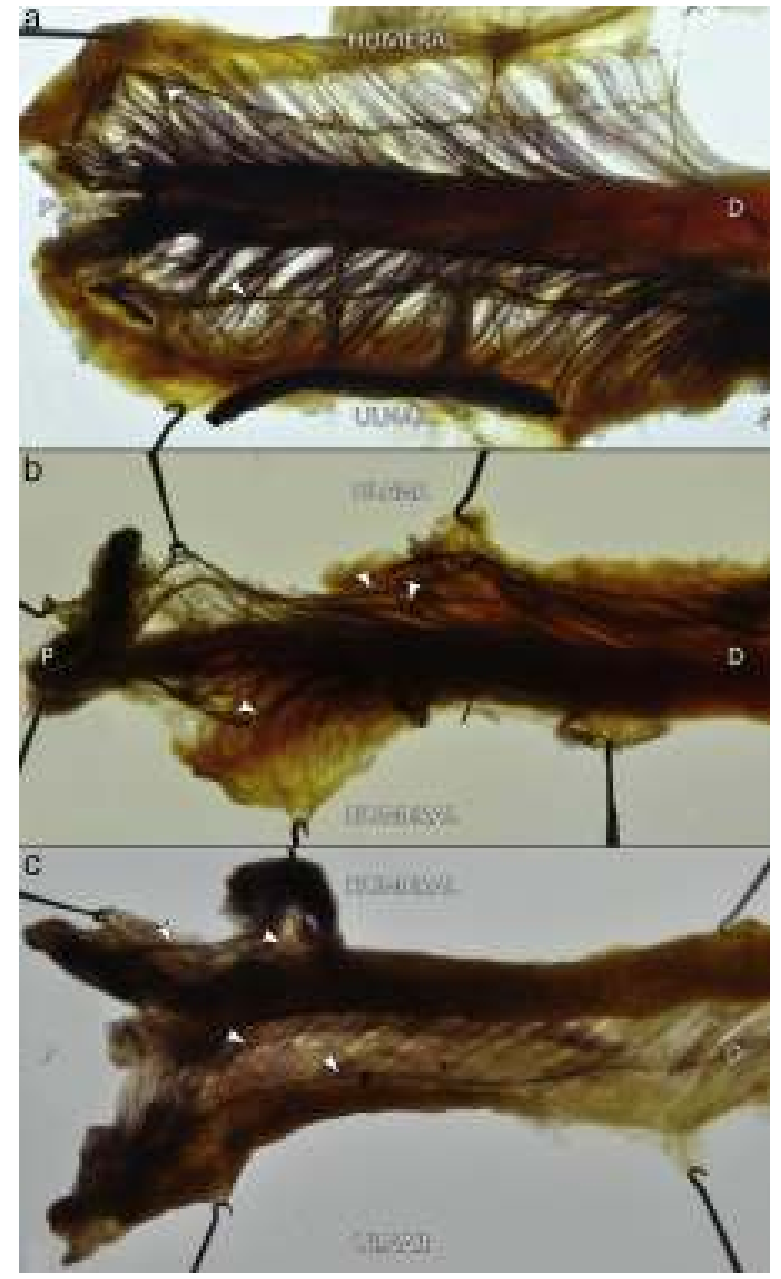

Figura 4 Especímenes teñidos según la técnica de Sihler. Se aprecian los distintos patrones de inervación intramuscular identificados: a) un ramo para cada cabeza; b) 2 ramos para la cabeza ulnar y uno para la humeral; c) 2 ramos para cada cabeza. D: extremo distal; P: extremo proximal; puntas de flecha: ramos intramusculares.

todos nuestros especímenes al tipo II implica que la partición quirúrgica respetando los ramos intramusculares es factible, tanto más dada la conformación bipennada del FCU, con un tendón central que actúa como elemento guía ${ }^{11}$.

La realización de transferencias tendinosas en el miembro superior cuenta ya con varias décadas de historia, a lo largo de las cuales los procedimientos se han ido refinando 
y diversificando. Las indicaciones más habituales de este tipo de intervención son aquellas lesiones nerviosas cuya reparación primaria fracasó, o que presentan pocas perspectivas de mejora, como las avulsiones radiculares ${ }^{12}$. También resultan útiles en el manejo de las secuelas de lesiones traumáticas con pérdida de masa muscular o tendinosa, en secuelas del síndrome compartimental y en la cirugía paliativa de problemas neurológicos de origen central como la parálisis cerebral o la lesión medular ${ }^{13}$. El desarrollo de variaciones técnicas que se puede derivar del conocimiento anatómico supone un valor añadido a estas técnicas, dada la gran diversidad de estos pacientes. Concretamente, la divisibilidad del músculo a transferir en 2 unidades neurofuncionales individualmente inervadas permite obtener de este 2 tendones transferibles a 2 músculos diferentes, los cuales pueden funcionar independientemente. De este modo, se optimizan los beneficios obtenidos de la transferencia: por ejemplo, al proporcionar un tendón más para transferir en pacientes con lesiones traumáticas complejas y extensas que limiten la disponibilidad de tendones donantes para hacer las transferencias clásicas. Otra ventaja aportada por el tendón adicional obtenido del FCU es que permite solventar la problemática fruto de algunas variaciones anatómicas: por ejemplo, la ausencia del palmaris longus (PL), que falta en hasta el $25 \%$ de individuos de algunas poblaciones, y cuyo tendón se transfiere frecuentemente al extensor pollicis longus en las parálisis radiales ${ }^{14}$. Finalmente, la preservación de al menos parte del FCU tal vez aliviaría las alteraciones biomecánicas secundarias al sacrificio del tendón del FCU en su totalidad. En la práctica, ya se conoce y se ha llevado a cabo la división del FCU y del FCR en algunos centros para realizar transferencias tendinosas ${ }^{15,16}$.

A pesar de lo dicho hasta el momento, cabe señalar que la aplicación clínica de la división del FCU (o de cualquier otro músculo similarmente compartimentado) en sus unidades neurofuncionales no acaba en las transferencias tendinosas. La presencia de múltiples pedículos vasculares (ninguno de los especímenes estudiados pertenecía al tipo I de la clasificación de Mathes-Nahai) asegura la viabilidad de ambas unidades al ser separadas, lo cual permite la realización de transferencias musculares libres funcionales, como reportaron Lim et al. en $2006^{17}$. La compartimentación de diversos músculos en el miembro superior ha sido estudiada ya por diversos autores; Chen et al. identificaron como mejores candidatos a la partición el FCU, el flexor carpi radialis, el extensor carpi ulnaris y el extensor carpi radialis brevis ${ }^{18}$. Algunos de estos grupos han llevado a cabo estudios anatómicos que describen y cuantifican las propiedades arquitectónicas de cada uno de los compartimentos, hecho fundamental para conocer la utilidad de cada músculo en particular ${ }^{19}$.

Por último, es importante destacar la vigencia de la tinción de Sihler modificada, que a pesar de los avances tecnológicos continúa siendo una herramienta difícil de igualar en el estudio morfológico del nervio periférico. La combinación de este método con la inyección vascular de látex, tal y como se ha hecho en nuestro trabajo, permite estudiar en el mismo espécimen la vascularización y la inervación, a diferencia de lo que ocurre en otros estudios de este tipo, habitualmente basados en técnicas radiológicas, incompatibles con la tinción y considerablemente más costosas.

\section{Conclusiones}

Según nuestro estudio todos los especímenes presentaban 2 unidades neurofuncionales claramente diferenciadas y superponibles a las 2 cabezas del FCU descritas clásicamente. Su inervación y vascularización independiente garantizan la funcionalidad y la viabilidad de ambas, incluso tras su separación quirúrgica. La configuración bipennada del músculo, con un tendón central que se origina muy proximalmente, permite una fácil división en el quirófano. La partición del tendón proporciona al cirujano un elemento transferible adicional y de propiedades biomecánicas específicas. De este modo, se obtienen 2 hemitendones procedentes de 2 unidades musculares que actúan como verdaderos músculos distintos. Esto multiplica las posibilidades técnicas disponibles en el ámbito de las transferencias tendinosas, puesto que aporta un elemento más para reparar o sustituir estructuras perdidas. Por otra parte, la divisibilidad del músculo en sí permite realizar procedimientos más complejos con sus unidades neurofuncionales, como transferencias libres funcionales $u$ otros procedimientos de cirugía reconstructiva relacionados.

\section{Conflicto de intereses}

Los autores declaran no tener ningún conflicto de intereses.

\section{Bibliografía}

1. English AW, Wolf SL, Segal RL. Compartmentalization of muscles and their motor nuclei: The partitioning hypothesis. Phys Ther. 1993;73:857-67.

2. Taylor GI, Minabe T. The angiosomes of the mammals and other vertebrates. Plast Reconst Surg. 1992;89:181-215.

3. Taylor GI, Caddy CM, Watterson PA, Crock JG. The venous territories (venosomes) of the human body: Experimental study and clinical implications. Reconstr Surg. 1990;86:185-213.

4. Taylor GI, Gianoutsos MP, Morris SF. The neurovascular territories of the skin and muscles: Anatomic study and clinical implications. Plast Reconstr Surg. 1994;94:1-36.

5. Lim AYT, Kumar VP, Hua J, Pereira BP, Pho RWH. The neuromuscular compartments of the flexor carpi ulnaris. Plast Reconstr Surg. 1999;103:1046-51.

6. Sihler C. Über muskelspindeln und intramuskuläre nervenendigungen bei Schlangen und Röschen. Arch Mikrosk Anat. 1895;46:709-23.

7. Won SY, Kim DH, Yang HM, Park JT, Kwak HH, Hu KS, et al. Clinical and anatomical approach using Sihler's staining technique (whole mount nerve stain). Anat Cell Biol. 2011;44:1-7.

8. Liu J, Kumar VP, Shen Y, Lau HK, Pereira BP, Pho RW. Modified Sihler's technique for studying the distribution of intramuscular nerve branches in mammalian skeletal muscle. Anat Rec. 1997;247:137-44.

9. Gülekon N, Peker T, Turgut HB, Anil A, Karaköse M. Qualitative comparison of anatomical microdissection. Sihler's staining and computerized reconstruction methods for visualizing intramuscular nerve branches. Surg Radiol Anat. 2007;29:373-8.

10. Mathes SJ, Nahai F. Classification of the vascular anatomy of the muscles: Experimental and clinical correlation. Plast Reconst Surg. 1981;67:177-87.

11. Lim AYT, Pereira BP, Kumar VP, de Coninck C, Taki C, Baudet J, et al. Intramuscular innervation of upper-limb skeletal muscles. Muscle Nerve. 2004;29:523-30.

12. Richards RR. Tendon transfers for failed nerve reconstruction. En: Mackinnon S, editor. Clinics in plastic surgery: 
Peripheral nerve surgery, 30. Philadelphia: Saunders; 2003. p. 223-46.

13. Sammer DM, Chung KC. Tendon transfers: Part I. Principles of transfer and transfers for radial nerve palsy. Plast Reconstr Surg. 2009;123:169e-77e.

14. Yammine K. Clinical prevalence of palmaris longus agenesis: A systematic review and meta-analysis. Clin Anat. 2013;26:709-18.

15. Lim AYT, Kumar VP, Pereira BP, Hua J. Independent function in a tendon transfer of the split flexor carpi ulnaris. Plast Reconstr Surg. 1999; 104:1739-43.

16. Lim AYT, Lahiri A, Pereira BP, Kumar VP, Tan LL. Independent function in a split flexor carpi radialis transfer. J Hand Surg (Am). 2004;29:28-31.
17. Lim AYT, Kumar VP, Sebastin SJ, Kapickis M. Split flexor carpi ulnaris transfer: A new functioning free muscle transfer with independent dual function. Plast Reconstr Surg. 2006;117:1927-32.

18. Chen G, Hua J, Liu AT, Zhang J, Lin Z, Dang RS, et al. Neurovascular details about forearm muscles: Applications in their clinical use in functional muscular transfer. Surg Radiol Anat. 2010;32:3-8.

19. Liu AT, Liu BL, Lu LX, Chen G, Yu DZ, Zhu L, et al. Architectural properties of the neuromuscular compartments in selected forearm skeletal muscles. J Anat. 2014;225: $12-8$. 